\title{
DESCENT-CYCLING IN SCHUBERT CALCULUS
}

\author{
ALLEN KNUTSON
}

\begin{abstract}
We prove two lemmata about Schubert calculus on generalized flag manifolds $\mathrm{G} / \mathrm{B}$, and in the case of the ordinary flag manifold $\mathrm{GL}_{n} / \mathrm{B}$ we interpret them combinatorially in terms of descents, and geometrically in terms of missing subspaces. One of them gives a symmetry of Schubert calculus that we christen descent-cycling. Computer experiment shows that these lemmata suffice to determine all of $\mathrm{GL}_{n}$ Schubert calculus through $n=5$, and $99.97 \%+$ at $n=6$. We use them to give a quick proof of Monk's rule. The lemmata also hold in equivariant ("double") Schubert calculus for Kac-Moody groups G.
\end{abstract}

\section{BACKGROUND ON SCHUBERT PROBLEMS}

Fix a pinning for a complex reductive Lie group G: a Borel subgroup $B$, an opposed Borel subgroup $B_{-}$, a Cartan subgroup $T=B \cap B_{-}$, the Weyl group $W=N(T) / T$, and $\mathrm{R}$ the Coxeter generators of $\mathrm{W}$. There is a famous basis (as a free abelian group) for the cohomology of G/B given by the Poincare duals of the closures of the $B_{-}$orbits on $G / B$; these are the Schubert classes $S_{w}:=\left[\overline{B_{-} w B / B}\right], w \in W$, and are indexed by the Weyl group.

(In this introduction we will only consider ordinary cohomology and the case of finitedimensional G. However, since the Schubert cycles $\overline{\mathrm{B} \_w \mathrm{~B} / \mathrm{B}}$ are T-invariant, they define elements not only of ordinary but of T-equivariant cohomology of G/B, and our results hold in that case also. In addition, our main arguments apply to the case of Kac-Moody G. Our references for equivariant cohomology of (possibly infinite-dimensional) G/B are $[\mathrm{G}, \mid \mathrm{KK}]$.)

The degree of the cohomology class $S_{w}$ is twice $l(w)$, the length of $w$ (as a minimal product of Coxeter generators from R). Define a Schubert problem as a triple $(u, v, w) \in$ $W^{3}$ such that $l(u)+l(v)+l(w)=\operatorname{dim}_{\mathbb{C}} G / B$. In this case we can consider the symmetric Schubert numbers

$$
c_{u v w}:=\int_{G / B} S_{u} S_{v} S_{w}
$$

which count the number of points in the intersection of three generic translates of Schubert cycles. Since this intersection is transverse (by a standard appeal to Kleiman's transversality theorem), and is of three complex subvarieties, the points are all counted with sign +1 and therefore the number is nonnegative. It is a famous open problem to compute this number combinatorially; the analogous problem for $G / P$ where $G=G L_{n}$ and $P$ is a maximal parabolic was solved first by the Littlewood-Richardson rule (or see [KT]).

Recall the Bruhat order on $W$ (due to Chevalley): $v>w$ if $v \in \overline{\mathrm{B}_{-} w \mathrm{~B} / \mathrm{B}}$. With this we can state our two lemmata:

Date: October 30, 2018.

This research was partially conducted for the Clay Mathematics Institute. 
Lemma 1. Let $(u, v, w)$ be a Schubert problem, and s a Coxeter generator. If us $>\mathrm{u}, v \mathrm{~s}>\boldsymbol{v}$, and $w s>w$, then $\mathrm{c}_{\mathrm{uvw}}=0$.

Lemma 2. Let $(u, v, w) \in W^{3}$ be a triple with $l(u)+l(v)+l(w)=\operatorname{dim}_{\mathbb{C}} G / B-1$, and $s$ a Coxeter generator. If $u s>u, v s>v$, and $w s>w$, then

$$
\mathrm{c}_{\mathfrak{u s}, v, w}=\mathrm{c}_{\mathfrak{u}, v s, w}=\mathrm{c}_{\mathfrak{u}, v, w s}
$$

In the case $G=G L_{n}(\mathbb{C}), W=S_{n}$, and $s$ is the transposition $i \leftrightarrow i+1$, the statement $u s>u$ says that $u(i)<u(i+1)$; one says that $u$ ascends in the $i$ th place. Otherwise if $u(i)>u(i+1)$ one says that $u$ descends in the $i$ th place, or that it has a descent there. For this reason we christen the symmetry of lemma 2 descent-cycling, and call these three problems dc-equivalent. Extending this relation by transitivity, we get a very powerful notion of equivalence for solving Schubert problems; in particular many Schubert problems are dc-equivalent to ones that fall to lemma 1, ones which we call dc-trivial.

Define a Grassmannian Schubert problem $(u, v, w)$ to be one in which $u, v$ each have only one descent, and in the same place, so named because the relevant integral can be performed on a Grassmannian; these Schubert problems are well-understood thanks to Littlewood-Richardson and other positive rules for their computation. It is worth pointing out that descent-cycling cannot be formulated in the context of Grassmannian problems alone;

1. descent-cycling a (nontrivial) Grassmannian Schubert problem always produces a non-Grassmannian Schubert problem;

2. Grassmannian problems from different Grassmannians (the single descent in different places) can be dc-equivalent.

In section 2 we define a graph whose vertices are Schubert problems and edges come from descent-cycling; by computer we were able to determine much about the structure of this graph in small examples. This we believe to be the main point of interest in the paper - that two such simple lemmata suffice to determine so many Schubert numbers.

It is our hope that this symmetry might help guide the search for a combinatorial formula for Schubert calculus; a rule generalizing Littlewood-Richardson (the case that $\pi$, $\rho$ each have only one descent, and in the same place) and manifestly invariant under descent-cycling would have very strong evidence for it 1

In section 3 we give the nearly-trivial proofs of the two lemmata, using standard properties of the (equivariant) BGG operators. We do this in terms of "Schubert structure constants" rather than symmetric Schubert numbers, which seems to be more appropriate for equivariant cohomology, and also gives results in the case of $\mathrm{G}$ a Kac-Moody group.

In the $\mathrm{GL}_{n}(\mathbb{C})$ case, there is an intuitive geometrical interpretation in terms of "reconstructing forgotten subspaces"; with this we can also say something about finding the actual flags in the intersection in synthetic-geometry terms, which we do in section 4.

In section [5]we prove Monk's rule via descent-cycling, to give an example of an interesting Schubert problem that falls to these techniques. It would be interesting to see if other known cases of $c_{u v w}=0,1$ (such as the Pieri rule $[\underline{S}]$ ) are consequences of descent-cycling.

\footnotetext{
${ }^{1}$ I circulated a preprint a year ago entitled "A conjectural rule for $\mathrm{GL}_{n}(\mathbb{C})$ Schubert calculus" in terms of puzzles, generalizing a Grassmannian theorem from [KT]. Alas, the rule conjectured there is not invariant under descent-cycling.
} 
We are thankful to Mark Haiman and Peter Magyar for useful comments.

\section{THE SCHUBERT PROBLEMS GRAPH, AND ITS STRUCTURE FOR SMALL GL $\mathrm{n}_{\mathfrak{n}}(\mathbb{C})$}

Let $\Gamma_{n}$ be the graph whose vertices are Schubert problems for $\mathrm{GL}_{n}(\mathbb{C})$, with edges between two Schubert problems that are related by cycling a single descent. Then the descent-cycling lemma 2 says that the symmetric Schubert number is constant on connected components of this graph 2 Recall that we define two Schubert problems to be dc-equivalent if they are in the same connected component, i.e., if one can be transformed into the other by a sequence of descent-cyclings. Also, we call a Schubert problem dctrivial if it falls to lemma 1, i.e. for some $(i, i+1)$ it has three ascents.

Example 1. We write a vertical bar to point out the descents, and a horizontal bar indicating to where we intend to cycle a descent. In the first line of figure 1 we descent-

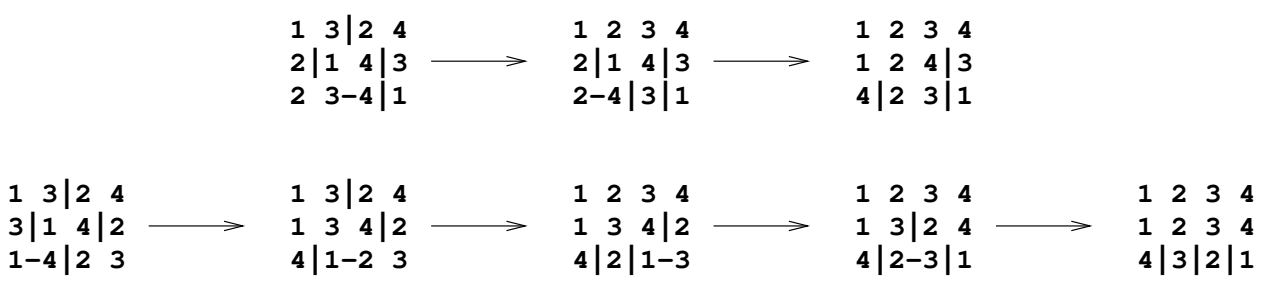

FIGURE 1. Using descent-cycling to simplify a Schubert problem, moving the vertical bars (marking descents) onto the horizontal ones.

cycle our way to a dc-trivial problem; this shows $c_{1324,2143,2341}=0$. In the second we show $(1324,3142,1423)$ dc-equivalent to $(1234,1234,4321)$, so $c_{1324,3142,1423}=c_{1234,1234,4321}$, which is in turn easily seen to be 1 . The reader may enjoy studying hands-on the properties of descent-cycling via the descent-cycling Java applet at

http://www.math. berkeley.edu/〜allenk/java/DCApplet.html

We established the following Facts by brute-force computation.

Fact 1. There are 35 Schubert problems for $\mathrm{GL}_{3}(\mathbb{C})$, of which 21 are not dc-trivial. All 21 live in the same connected component of $\Gamma_{3}$, which is pictured in figure 2

Fact 2. Let $\mathrm{n} \leq 5$, and $(\pi, \rho, \sigma)$ be a Schubert problem in dimension $\mathrm{n}$. Then the symmetric Schubert number $\mathrm{c}_{\pi \rho \sigma}$ equals zero if and only if $(\pi, \rho, \sigma)$ is dc-equivalent to a dc-trivial problem. Otherwise, $(\pi, \rho, \sigma)$ is dc-equivalent to $\left(i d, i d, w_{0}\right)$ (where id denotes the identity permutation and $w_{0}$ the long word), and therefore the symmetric Schubert number $\mathrm{c}_{\pi \rho \sigma}$ equals one. Put another way, there is exactly one non-dc-trivial component of the Schubert problem graph for each $\mathrm{n} \leq 5$.

In particular, the two lemmata (and the trivial calculation $c_{i d, i d, w_{0}}=1$ ) suffice to completely determine Schubert calculus for $\mathrm{GL}_{n}(\mathbb{C})$ through $n=5$. We know a priori that this connectedness cannot continue at $n=6$, because the nonzero symmetric Schubert numbers are sometimes 2. (All symmetric Schubert numbers in this paper were computed with the Maple package [ACE].)

\footnotetext{
${ }^{2}$ Probably a graph structure is not quite the right one to use for this, since the natural concept of "edge" here connects three, not two, vertices.
} 


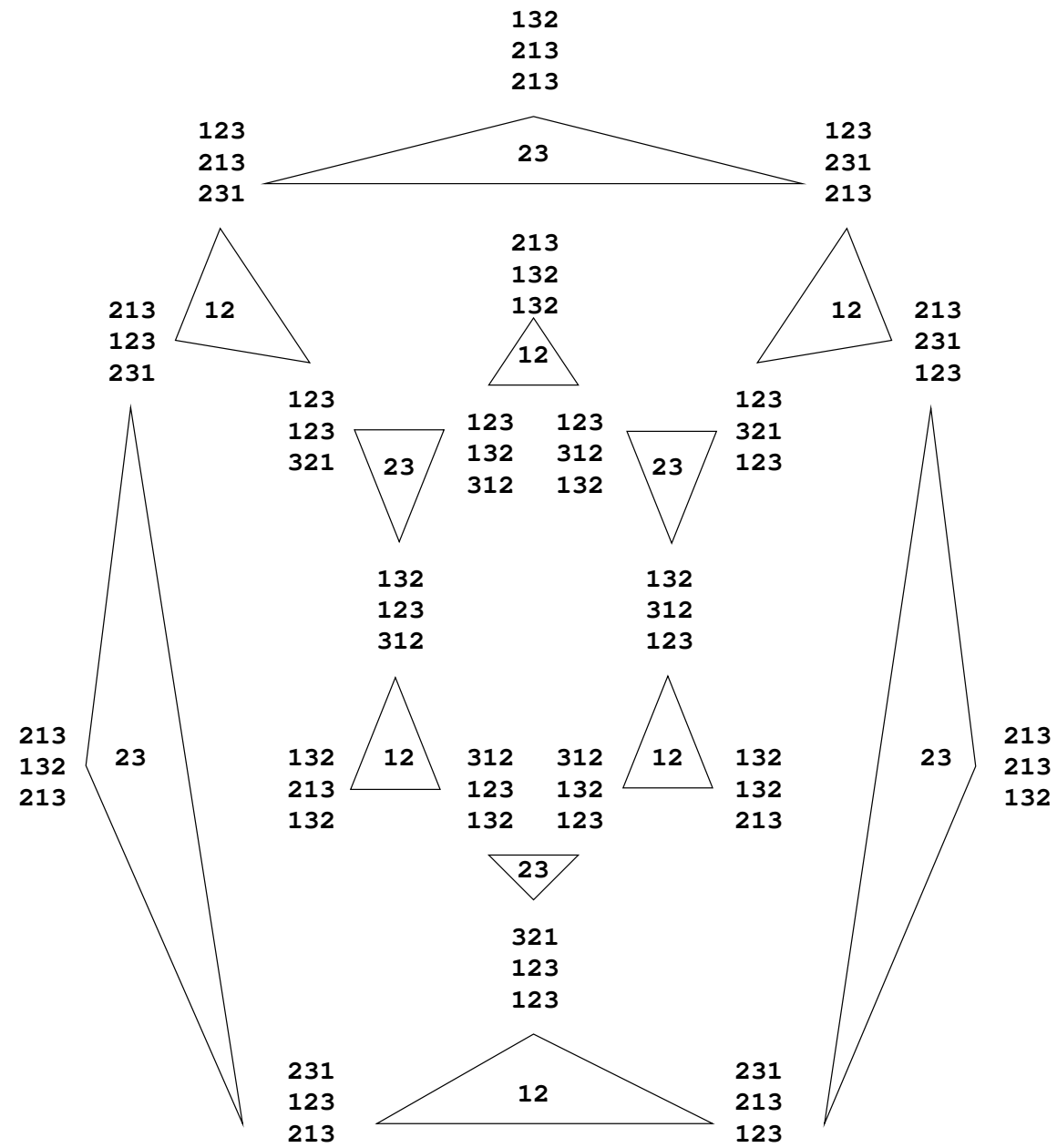

FIGURE 2. The non-dc-trivial component of $\Gamma_{3}$, drawn to make its $S_{3}$ symmetry manifest. The edges, which always come in sets of three, are drawn as triangles and labeled with the column where descents are being cycled. Note that not all vertices have degree 4; one cannot descent-cycle in a column which has two descents.

Fact 3. The graph $\Gamma_{6}$ has $8,881,334$ vertices, of which all but $2,351,475$ are dc-trivial. Throwing out the components with dc-trivial vertices we are left with 145 components comprising 411,582 vertices. The lion's share of those vertices, 409,023, are dc-equivalent to the easy case ( $\left.i d, i d, w_{0}\right)$, leaving 2559 cases (less than $0.03 \%$ ) not succumbing to dc-equivalence/dc-triviality arguments.

Of the remaining 144 components, only one contains a Grassmannian Schubert problem, so in some sense the Littlewood-Richardson rule doesn't help much. (In fact this is the only one with any Grassmannian permutations, so a Schubert-times-Schur rule wouldn't help much either.)

Exactly one of these components has intersection number zero (despite containing no dc-trivial Schubert problems); one element of it is $(231645,231645,326154)$. There are also 48 components of size one, i.e. Schubert problems that admit no descent-cycling whatever; one example is $(214365,154326,321654)$. 
These computations were done in C and took 2.5 minutes on a Pentium 300 . The limiting factor was that they just barely fit in 64 megabytes of RAM, putting the $n=7$ case (which is roughly $7^{3}=343$ times bigger) out of reach without new ideas.

It seems likely that as $n$ increases, the fraction of $G L_{n}(\mathbb{C})$ Schubert problems having no place with three ascents (and so falling to lemma 1 alone) goes to 0 . We did not pursue this.

It was very tempting to believe that a vanishing Schubert number could always be "blamed" on dc-equivalence to a dc-trivial problem, and it was very sad to find the lone component in $\Gamma_{6}$ that belies this. Mark Haiman pointed out a "stabilization" map $\Gamma_{n-1} \hookrightarrow \Gamma_{n}$ taking $(u, v, w) \mapsto(u n, v n, 1(w+1))$ (where $w+1$ means to add 1 to all elements of $w$ ). (For example, $(2143,1243,3214) \mapsto(21435,12435,14325)$.) Question: can distinct components become connected under this map (possibly connecting the rogue component in $\Gamma_{6}$ to a dc-trivial problem)?

\section{PROOFS OF THE LEMMATA}

The statements in this section are slightly different from those in the introduction, in that they are phrased in terms of structure constants $c_{u v}^{w}$, rather than symmetric Schubert numbers $c_{u v w}$. We first remind the reader of the partial relation between these and explain why we switch to the less-symmetric formulation.

In ordinary cohomology of G/B (G finite dimensional), we have the Poincaré-pairing duality relation

$$
\int_{\mathrm{G} / \mathrm{B}} \mathrm{S}_{\mathrm{u}} \mathrm{S}_{v}=\delta_{\mathrm{u}, w_{0} v}
$$

where $w_{0}$ is the long element of the Weyl group, and $\delta$ is the Kronecker delta. (Note that the duality discussed here is in the sense of dual bases, and not Poincare duality!)

One way to see this is to realize the class $S_{v}$ not by the Schubert cycle $\overline{\mathrm{B} \_v \mathrm{~B}}$ but the opposite Schubert cycle $\overline{\mathrm{B} w_{0} v \mathrm{~B}}=w_{0} \overline{\mathrm{B}_{-} v \mathrm{~B}}$. Since $w_{0}$ is connected to the identity in $\mathrm{G}$, these two cycles define the same element of cohomology.

From this we derive that if

$$
\mathrm{S}_{\mathrm{u}} \mathrm{S}_{v}=\sum_{w} \mathrm{c}_{\mathrm{uv}}^{w} \mathrm{~S}_{w}, \quad \text { then } \quad \mathrm{c}_{\mathrm{uv}}^{w}=\mathrm{c}_{\mathrm{u}, v, w_{0} w}
$$

So in ordinary cohomology, one can work instead with these Schubert structure constants, although our results from the first section are prettier to state symmetrically. However, in T-equivariant cohomology the dual basis to the Schubert basis is not once again the Schubert basis (essentially because $w_{0}$ is not connected to the identity through $\mathrm{T}$ invariant maps of $\mathrm{G} / \mathrm{B}$ ) and these two concepts part ways.

In $[G]$ a certain positivity result was proven for the equivariant $c_{u v}^{w} \in H_{T}^{*}$ (which must be carefully stated, insofar as these are polynomials not numbers). This implies a much weaker positivity result for the $c_{u v w}$, and so it seems more interesting to prove results about the structure constants. 
Also, in the case of $\mathrm{G}$ an infinite-dimensional Kac-Moody group, one cannot so blithely do integrals on $G / B$, and the $c_{u v}^{w}$ are the only concept that makes sense. This concludes the advertisement for Schubert structure constants over symmetric Schubert numbers 3

Note that the condition we gave in section 1 for a "Schubert problem" corresponds to $l(w)=l(u)+l(v)$, which seems a reasonable thing to ask since cohomology is a graded ring. But we will not insist on this in what follows because, in equivariant cohomology, the structure constants can be nonzero even if one only has $l(w) \leq l(u)+l(v)$. (We only imposed this condition before to keep the graph of Schubert problems a reasonable size.)

Let $s \in R$ be a simple reflection, and $P_{s}=\overline{B s B} \leq G$ be the corresponding minimal parabolic. Let $p_{s}: G / B \rightarrow G / P_{s}$ be the corresponding projection (which is G-equivariant and therefore T-equivariant); composing pushforward with pullback defines a degree -2 endomorphism $\partial_{s}$ on $\mathrm{H}_{\mathrm{T}}^{*}(\mathrm{G} / \mathrm{B})$ (first introduced in [BGG] and [D], stated nonequivariantly, although equivariant K-theory is implicit in [D]).

We refer to $[\mathrm{KK}]$ for the four properties we need of these BGG operators $\partial_{s}$ :

1. If $w s>w$, then $\partial_{s} S_{w}=0$.

2. If $w s<w$, then $\partial_{s} S_{w}=S_{w s}$.

3. With respect to a certain natural action of $W$ on $\mathrm{H}_{\mathrm{T}}^{*}(\mathrm{G} / \mathrm{B}), \partial_{\mathrm{s}}$ is a twisted derivation:

$$
\partial_{s}(\alpha \beta)=\alpha \partial_{s}(\beta)+\partial_{s}(\alpha)(s \cdot \beta) .
$$

(We won't need to understand this action of $W$.)

4. If $s_{1} s_{2} \ldots s_{l}=r_{1} r_{2} \ldots r_{l}$ are two reduced expressions for a Weyl group element $w$, then $\partial_{s_{1}} \ldots \partial_{s_{l}}=\partial_{r_{1}} \ldots \partial_{r_{l}}$, and so we have a well-defined operator $\partial_{w}$.

Since the proofs of both lemmata have much in common, we gather them into a single proposition.

Proposition 1. Let $(\mathfrak{u}, v, w) \in W^{3}$, and s a simple reflection, such that $u s>\mathfrak{u}$ but $w s<w$.

- If $v s>v$, then $\mathrm{c}_{\mathrm{uv}}^{w}=0$.

- If $v s<v$, then $\mathbf{c}_{\mathfrak{u} v}^{w}=c_{\mathfrak{u}, v s}^{w s}$.

Proof. The main formula we need is

$$
c_{x y}^{z}=\text { coefficient of } S_{1} \text { in } \partial_{z}\left(S_{x} S_{y}\right)
$$

which follows easily from the properties stated of the BGG operators.

Since $w s<w$, we have

$$
\partial_{w}\left(S_{u} S_{v}\right)=\partial_{w s} \partial_{s}\left(S_{u} S_{v}\right)=\partial_{w s}\left(S_{u} \partial_{s}\left(S_{v}\right)+\partial\left(S_{u}\right)\left(s \cdot S_{v}\right)\right)=\partial_{w s}\left(S_{u} \partial_{s}\left(S_{v}\right)\right)
$$

this last because by the $u s>\mathfrak{u}$ assumption, $\partial_{s}$ annihilates $S_{u}$.

If $v s>v$, then $\partial_{s}$ annihilates $S_{v}$ too, and the RHS is zero. Combining that with the formula for $\mathrm{c}_{x y}^{z}$ gives the first result.

If $v s<v$, then the RHS is $\partial_{w s}\left(S_{u} S_{v s}\right)$, and two applications of the formula for $c_{x y}^{z}$ give the second result.

\footnotetext{
${ }^{3}$ In fact almost all work on Littlewood-Richardson is in terms of the structure constants; see [KT] for a discussion of this.
} 
The conditions on $w s$ versus $w$ in this proposition are backwards from how they were in lemmata 1 and 2, that's because of the multiplication by $w_{0}$ in comparing Schubert structure constants with symmetric Schubert numbers. With this in mind the two lemmata follow.

\section{A GEOMETRICAL INTERPRETATION}

For $w \in \mathrm{W}$, let $\mathrm{D}_{w}:=\overline{\mathrm{G} \cdot(w \mathrm{~B}, \mathrm{~B})} \subseteq(\mathrm{G} / \mathrm{B})^{2}$. Given a simple reflection $\mathrm{s} \in \mathrm{R}$, let $\mathrm{P}_{\mathrm{s}}$ again be the corresponding minimal parabolic $\overline{\mathrm{BsB}}$, and consider the composite map $\mathrm{D}_{w} \hookrightarrow(\mathrm{G} / \mathrm{B})^{2} \rightarrow \mathrm{G} / \mathrm{P}_{\mathrm{s}} \times \mathrm{G} / \mathrm{B}$.

Lemma 3. Let $w \in W, s \in R, P=\overline{B s B}$. The fibers of $D_{w} \hookrightarrow(G / B)^{2} \rightarrow G / P_{s} \times G / B$ are

- $\mathbb{C P}^{1}$ 's if ws $<w$

- single points (generically), if $w \mathrm{~s}>w$.

Proof. We reduce to the well-studied case (see [D]) of a single flag manifold. Let $X:=$ $\mathrm{G} / \mathrm{B} \times\{\mathrm{B} / \mathrm{B}\}$; since $\mathrm{G} \cdot \mathrm{X}=\mathrm{G} / \mathrm{B} \times \mathrm{G} / \mathrm{B}$ it suffices to consider the map $\mathrm{D}_{w} \cap \mathrm{X} \rightarrow \mathrm{G} / \mathrm{P}_{\mathrm{s}} \times\{\mathrm{B} / \mathrm{B}\}$. And $D_{w} \cap X=\overline{B w B} / B \times\{B / B\}$, so (omitting the $\{B / B\}$ ) we're studying the fibers of the composite $\overline{\mathrm{B} w \mathrm{~B} / \mathrm{B}} \rightarrow \mathrm{G} / \mathrm{B} \rightarrow \mathrm{G} / \mathrm{P}_{\mathrm{s}}$, as already done in [D].

In the case of $G=G L_{n}(\mathbb{C}), D_{w}$ is the variety of pairs of flags $(F, G)$ in $\mathbb{C}^{n}$ such that " $F$ is $w$-close or closer to $G^{\prime \prime}$. In this case, the generators $R$ correspond 1:1 to the subspaces in a flag (other than the zero subspace and the whole space), and the map $G / B \rightarrow G / P_{s}$ corresponds to "forgetting" the subspace. Then we can interpret the lemma in very familiar terms:

Corollary. Let $w \in S_{n}, i \in 2 \ldots n-1$. Let $\mathrm{F}, \mathrm{G}$ be two flags in $\mathbb{C}^{\mathrm{n}}$ such that $\mathrm{F}$ is $w$-close or closer to $\mathrm{G}$. Let $\mathrm{F}^{\prime}$ be the partial flag obtained by forgetting $\mathrm{F}^{\prime} \mathrm{s} i$-dimensional subspace. Can we reconstruct $\mathrm{F}$ knowing only $\mathrm{F}^{\prime}, \mathrm{G}$, and $w$ ?

- If $w$ ascends at $(i, i+1)$, there is no hope - any $i$-dimensional space in between $F_{i-1}$ and $F_{i+1}$ will do.

- If $w$ descends at ( $i, i+1)$, then (for a Zariski-open set of such $\mathrm{G}$ ) the subspace $\mathrm{F}_{i}$ is uniquely determined.

Another way to interpret this is that if $w$ does not descend at $(i, i+1)$, then $G$ "does not care" what $F_{i}$ is used (to get $F w$-close to $G$ ). Conversely, if $w$ does descend there, then $G$ "usually insists" on a particular $F_{i}$, when presented with the rest of $F$.

Proof of lemma 1 Let $\{\mathrm{F}\}$ be the set of flags in relative position $u$ to $A, v$ to $B$, and $w$ to $C$ where $A, B, C$ are three flags in generic relative position. Then by codimension count (and the usual appeal to Kleiman's transversality theorem) the set $\{\mathrm{F}\}$ is finite. However, since none of $A, B, C$ care what $F_{i}$ is (since by assumption none of them have a descent at $(i, i+1))$, the set $\{F\}$ is a union of $\mathbb{C P} \mathbb{P}^{1}$ s. These two facts are only compatible if $\{F\}$ is empty.

Proof of lemma 2 Let $\{\mathrm{F}\}$ be the set of flags in relative position $u$ to $A, v$ to $B$, and $w$ to $C$ where $A, B, C$ are three flags in generic relative position. Then as in the previous proof, the set $\{\mathrm{F}\}$ is a union of $\mathbb{C P}^{1}$ s, reflecting the ambiguity in $\mathrm{F}_{i}$. If we change one of $u, v, w$ to 
have a descent at $(i, i+1)$, each of these $\mathbb{C P}^{1}$ s is cut down to a single point. But it doesn't matter which of $u, v, w$ gets this new descent.

This geometric description of descent-cycling suggests that additional symmetries may come from forgetting multiple subspaces at a time. It appears, though, that all of these are implied by the single-subspace case.

One application of this geometric description is to actually locate the flag satisfying the desired intersection conditions, in the case that $(\pi, \rho, \sigma)$ is dc-equivalent to the easy case $\left(\mathrm{id}, \mathrm{id}, w_{0}\right)$. We illustrate this in the case of the Schubert problem $(132,213,213)$, which we can descent-cycle to $(123,213,231)$, and from there to $(123,123,321)$. Working from the end, the unique $F$ satisfying $(123,123,321)$ is $F_{1}=C_{1}, F_{2}=C_{2}$. When we cycle the descent in the $(1,2)$ column, we have to replace $F_{1}=C_{1}$ by $F_{1}=B_{2} \cap C_{2}$. Then when we cycle the descent in the $(2,3)$ column we have to replace $F_{2}=C_{2}$ by $F_{2}=A_{1} \oplus\left(B_{2} \cap C_{2}\right)$.

There is an alternate way to prove the vanishing condition in proposition 1 cohomologically, involving the projection $G / B \rightarrow G / P_{s}$. A Schubert class $S_{u}$ is in the image of the pullback of $\mathrm{H}_{\mathrm{T}}^{*}\left(\mathrm{G} / \mathrm{P}_{\mathrm{s}}\right)$ if and only if $u s>\mathrm{u}$. Since this pullback is a ring homomorphism, the product of two pulled-back classes is also in this image, and cannot involve any $S_{w}$ with $w s<w$.

\section{MONK'S RULE}

Monk's rule $[M]$ is concerned with the case of $\mathrm{GL}_{n}(\mathbb{C})$ Schubert problems in which $\rho$ is a simple reflection $s_{i}=(i \leftrightarrow i+1)$.

Theorem (Monk's rule). Let $\sigma w_{0}$ cover $\pi$ in the Bruhat order; i.e. $\sigma$ is $\pi$ with each number $j$ replaced by $(n+1)-j$, and two numbers inverted in $\pi w_{0}$ have been put back in correct order, decreasing the number of inversions by exactly one. Then $\mathrm{c}_{\pi, s_{i}, \sigma}=1$ if the numbers switched straddled the position between $i$ and $i+1$, whereas $c_{\pi, s_{i}, \sigma}=0$ if the numbers switched were both physically on one side of $(i, i+1)$.

(Some may object that Monk's rule says more - that $c_{\pi, s_{i}, \sigma}=0$ unless $\sigma w_{0}$ covers $\pi-$ but we prefer to see this as a more general property of symmetric Schubert numbers, that if $\pi, \rho$ are not less than $\sigma w_{0}$ in the Bruhat order, then $c_{\pi \rho \sigma}=0$.)

For example, let $\pi=34152, i=2$. Then $\pi w_{0}=32514$, which covers $23514,31524,32154$, 32415. But only 31524 involves switching a number in the first 2 places with a number in the last 5-2 places. So $c_{34152, s_{i}, 31524}=1$, but $c_{34152, s_{i}, 23514}=c_{34152, s_{i}, 32154}=c_{34152, s_{i}, 32415}=0$.

Theorem 1. Let $\mathrm{f}: \mathrm{V}\left(\Gamma_{\mathrm{n}}\right) \rightarrow \mathbb{Z}$ be a functional on the set of Schubert problems. If $\mathrm{f}$ satisfies the properties

1. $f$ is invariant under descent-cycling (i.e. is constant on components)

2. $f=0$ on dc-trivial Schubert problems

3. $\mathrm{f}\left(\mathrm{id}, \mathrm{id}, w_{0}\right)=1$

then $\mathrm{f}$ obeys Monk's rule, i.e. $\mathrm{f}\left(\pi, s_{i}, \sigma\right)=0$ or 1 according to the criterion of Monk's rule.

We first prove a lemma:

Lemma 4. Let $\mathrm{f}$ satisfy the conditions of theorem 1 and $\pi, \sigma \in S_{\mathfrak{n}}$ such that $l(\pi)+l(\sigma)=\left(\begin{array}{c}\mathfrak{n} \\ 2\end{array}\right)$. Then if $\pi=\sigma w_{0}$, we have $\mathrm{f}(\pi, \mathrm{id}, \sigma)=1$, and otherwise $\mathrm{f}(\pi, \mathrm{id}, \sigma)=0$. 
Proof. Since the second argument has no descents, any place $(i, i+1)$ that $\pi$ has a descent and $\sigma$ does not gives us an opportunity to cycle a descent from the first argument to the third, replacing $\pi \mapsto s_{i} \pi, \sigma \mapsto s_{i} \sigma$. This modification keeps the sum of the lengths $=\left(\begin{array}{l}n \\ 2\end{array}\right)$ and neither causes nor breaks the condition $\pi=\sigma w_{0}$. So we can reduce to the case that any descent in $\pi$ occurs at a descent of $\sigma$.

If $\pi=\sigma w_{0}$ : then each ascent in $\sigma$ occurs at a descent of $\pi$. By our reduction above, this means that $\sigma$ has no ascents. So we're looking at $f\left(i d, i d, w_{0}\right)$ which by assumption is 1 .

Conversely if $f(\pi, i d, \sigma) \neq 0$ : then no column $(i, i+1)$ has three ascents (the Schubert problem $(\pi, i d, \sigma)$ is not dc-trivial). By our reduction, this means that $\sigma$ has no ascents. So $\sigma=w_{0}$. By the assumption on the total length, $\pi=i d$, so $\pi=\sigma w_{0}$ as desired.

Proof of theorem 1 Let $\sigma$ be $\pi w_{0}$ with the numbers in the $j$ th and $k$ th positions switched, decreasing the number of inversions by exactly one (and so that $j<k, \sigma(j)<\sigma(k)$ ). In particular every number in $\sigma$ physically between the $j$ th and kth positions is not numerically between $\sigma(j)$ and $\sigma(k)$. We want to show that $f\left(\pi, s_{i}, \sigma\right)=0$ unless $j \leq i<k$, in which case $f\left(\pi, s_{i}, \sigma\right)=1$.

First, we treat the case $k=j+1$. If $j=i, k=i+1$, then neither $\pi$ nor $\sigma$ has a descent at $(i, i+1)$. So we can cycle the descent from the second argument of $f$ into the third, making them $\left(\pi, i d, \pi w_{0}\right)$. Now lemma团 tells us that this 1 .

Whereas if $j, k \leq i$ or $j, k \geq i+1$ (still in the case $k=j+1$ ), then none of $\pi$, $i d$, or $\sigma$ have a descent at $(j, k)$, and therefore $f$ vanishes as it's supposed to.

Now take the case $k>j+1$. Then since $\sigma$ has only one fewer inversion than $\pi w_{0}, \sigma$ must have the same descent-pattern as $\pi w_{0}$. Now we reduce (much as in lemma 4 ) by cycling descents between the first and third arguments, in order to move the positions $j$ and $k$ closer together.

We can do this descent-cycling in the $(j, j+1)$ column as long as $j \neq i$, and the $(k-1, k)$ column as long as $k \neq i+1$. If $j, k$ are both on the same side of the $(i, i+1)$ divide, they can be brought next to each other (by e.g. just moving one of them). If $j, k$ are on opposite sides of the divide, we can at least get $j$ up to $i$, and $k$ down to $i+1$. Either way we reduce to the $k=j+1$ case and therefore get the same answer as Monk's rule.

In particular, this gives an explicit sequence of descent-cyclings to turn a Monk's rule problem into $\left(i d, i d, w_{0}\right)$. So in principle one can reverse the steps and construct the flag in the intersection of these three Schubert varieties, as an expression in the lattice of subspaces.

There are other special cases known for symmetric Schubert numbers where the answer is 0 or 1, mostly notably the Pieri rule (see [R, $[\mathbf{S}]$ ); it would be interesting to see if they too are consequences of descent-cycling. Probably the best version of this would be a "descent-cycling normal form" for Schubert problems, and an effective way to test whether a Schubert problem is dc-equivalent to $\left(\mathfrak{i d}, \mathfrak{i d}, w_{0}\right)$.

\section{SYNTHETICITY VS. $\mathrm{c}=1$ QUESTIONS}

Recall that given a Schubert problem $\mathrm{P}=(\pi, \rho, \sigma)$, and three generically situated flags $A, B, C$, one can think of the symmetric Schubert number $c_{\pi \rho \sigma}$ as the number of flags $F$ such that $F$ is $\pi$-close to $A, \rho$-close to $B$, and $\sigma$-close to $C$. 
The Schubert problem $\left(i d, i d, w_{0}\right)$ is then easily seen to have symmetric Schubert number one; to be id-close to $A$ or $B$ is no condition at all, and to be $w_{0}$-close to $C$ requires $F_{i}=C_{i}$ for all $i=1, \ldots, n$.

Consider the following four statements one might make about a Schubert problem $\mathrm{P}=$ $(\pi, \rho, \sigma)$ :

- dc-easiness: $P$ is dc-equivalent to the Schubert problem ( $i d, i d, w_{0}$ )

- partial syntheticity: there is a flag in the free modular lattice on three flags $A, B, C$ satisfying $P$

- full syntheticity: every flag satisfying $P$ is in the free modular lattice on three flags $A, B, C$

- $c=1$ : the symmetric Schubert number $c_{\pi \rho \sigma}=1$.

So $P$ dc-easy implies $P$ fully synthetic and $c_{P}=1$. The other possible implications seem to be unknown.

Question. Does $c=1$ imply partial (and thus full) syntheticity? This would seem to be a Galois theory argument, with "synthetic" the analogue of "rational."

Question. Does $\mathrm{P}$ partially synthetic imply $\mathrm{P}$ stably dc-easy? If the flag $\mathrm{F}$ is a synthetic solution to the Schubert problem $P$, i.e. $F_{i}$ is a lattice word in $A, B, C$ for each $i$, perhaps there is an algorithm to "simplify" the "worst" subspace in P using descent-cycling, with the only unsimplifiable subspaces being those in $A, B, C$. In particular, this would say that $P$ partially synthetic implies $P$ fully synthetic.

\section{REFERENCES}

[ACE] S. Veigneau, ACE, an Algebraic Combinatorics Environment for the computer algebra system MAPLE, User's Reference Manual, Version 3.0, IGM 98-11, Université de Marne-la-Vallée, 1998.

[BGG] I. N. Bernstein, I. M. Gel'fand, S. I. Gel'fand, Schubert cells and the cohomology of a flag space, Uspekhi Mat. Nauk 28 (1973), no. 3 (171), 3-26.

[D] Michel Demazure, Désingularisation des variétés de Schubert généralisées, Ann. Sci. École Norm. Sup. (4) 7 (1974), 53-88.

[KK] Bertram Kostant, Shrawan Kumar, The nil Hecke ring and cohomology of G/P for a Kac-Moody group G, Advances in Mathematics 62 (1986), no. 3, 187-237.

[KT] Allen Knutson, Terence Tao, Puzzles, (equivariant) cohomology of Grassmannians, and the MolevSagan rule, in preparation.

[M] D. Monk, The geometry of flag manifolds, Proc. London Math. Soc. (3) 91959 253-286.

[G] William Graham, Positivity in equivariant Schubert calculus, math. AG/9908172

[R] Shawn Robinson, The equivariant Pieri rule, in preparation.

[S] Frank Sottile, Pieri's formula via explicit rational equivalence, Canad. J. Math. 49 (1997), no. 6, 12811298.

E-mail address: allenk@math . berkeley.edu 